\title{
A transgenic zebrafish for discovering viruses and studying host-pathogen interactions
}

Balla, K.M., Rice, M.C., Gagnon, J.A., and Elde, N.C. Curr. Biol. 30, 2092-2103.E5 https://doi.org/10.1016/j.cub.2020.04.031 (2020).

Viruses are everywhere, ever in search of the host organisms they need to infect in order to replicate. Those hosts, however, don't always appreciate the company, mounting immune responses to keep invaders in check. Sometimes that leads to severe illness, but not always-hosts can be infected without displaying any clinical symptoms. Such viruses carry on undetected, though they may in fact be influencing their hosts in subtle ways.

As metagenomics technologies advance that enable detection of novel microbes in the environment, such asymptomatic infections are increasingly turning up in laboratory animals, with potentially confounding effects on various phenotypes that researchers are growing increasingly aware of. Yet there is still much to learn about how animal hosts and the pathogens that infect them interact and co-evolve. A new paper from Nels Elde's lab at the University of Utah provides takes that work to the level of a whole organism: the zebrafish.

Elde's lab specializes in the evolution of host-pathogen conflict, but much of their work has been done in mammalian cell cultures, says postdoctoral research Keir Balla. Those are powerful systems, but they can lack the larger biological context that comes from studying a whole animal. To start exploring that context, they've turned to the zebrafish. "It's such a versatile model of vertebrate biology, but it is somewhat under-explored in terms of infection biology," says Balla.

Previously, Balla had studied natural infections and immunity in another whole organism: C elegans. With the worms, he could carefully control exposure to different pathogens of interest while keeping other variables in check. "We know everything that the worm is interacting with in the laboratory environment," says Balla, "That's much harder to control for fish."

That may be a disadvantage for some areas of research, but not necessarily for studying host-pathogen interactions. "There were already infectious microbesin particular viruses-hanging out in the laboratory environment that people weren't aware of," he says. "That turned into the opportunity to explore this messy environment, even within the laboratory, and to find some interesting host-pathogen interactions."

But first, they needed a way to detect any infections occurring in their zebrafish.

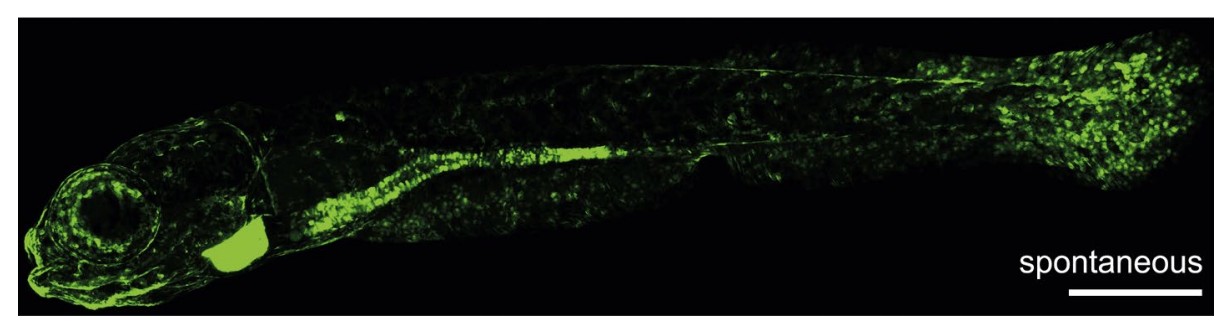

Virus alert! A larval zebrafish fluoresces green in response to interferon. Reprinted with permission from Balla (2020) Elsevier, Inc.

You can't always distinguish an infected fish by appearance says Balla, and unlike for bacteria and fungi where there are means to detect non-specific infections, no such general markers existed for detecting unknown viruses.

They decided to make the immune system itself the marker, generating transgenic zebrafish that express green fluorescent protein (GFP) in response to the release of interferon. The interferon system, Balla explains, is a first line of immune defense for all jawed vertebrates: when pattern recognition molecules detect a potential pathogen, interferon proteins are produced that notify the rest of the immune system to the presence of the invader. "We use this alarm system as the main signaling platform," says Balla.

While Balla and his colleagues were confirming that their isg15:GFP transgenic fish indeed expressed GFP as intended when interferon-expressing plasmids were injected into them, the team noticed that untreated control fish were also taking on a greenish glow, particularly in environment-exposed tissues such as the intestine and epithelial surfaces. Sequencing of RNA sampled from various GFP-positive, GFP-negative, and wildtype fish revealed the presence of a novel zebrafish picornavirus, ZfPV.

ZfPV turned out to be endemic to the Utah zebrafish facility-and it also turned up in 92 existing RNA-seq datasets from research facilities across the world that had been deposited in the NIH Sequence Read Archive. Among those, the researchers detected several biological replicates with gene expression patterns that varied with the presence of ZfPV. That observation adds to the growing list of asymptomatic infections in various laboratory species that can potentially confound phenotypes without causing overt illness.
In their current paper, Balla and his colleagues then used the zebrafish to experimentally study viral transmission, showing that standard egg bleaching protocols can prevent naturally occurring $\mathrm{ZfPV}$ infections and that infected larvae can horizontally transmit the virus to any uninfected individuals with which they are co-housed.

Balla is now using the new zebrafish to further explore the nature of host-pathogen interactions, with plans to expand to other environments outside the lab, such as pet shops. "We know there are a lot of close relatives of zebrafish that are commonly traded," he says, offering the opportunity to study a wider range of potential pathogens and how those might adapt from one host species to another.

More broadly, the results underscore the importance of the microbiome and the microbial interactions that contribute to an organism's development and health. "By tapping into all of these interactions that are important for zebrafish biology, I think this just adds a nice layer to that thinking," he says.

Though any viruses that manage to evade the interferon system will remain undetected with at least this particular model, the isg15:GFP transgenic zebrafish can serve two purposes, acting as sentinels to the presence of novel viruses while offering researchers a means to visualize host immune response to those same infections. "Because we use the host response as a way to find the virus, we've merged those concepts," says Balla, connecting viral discovery to host-pathogen interactions in one whole organism.

\section{Ellen P. Neff}

Published online: 15 June 2020 https://doi.org/10.1038/s41684-020-0581-6 